\title{
Identifying motor imagery activities in brain computer interfaces based on the intelligent selection of most informative timeframe
}

\author{
Hamidreza Abbaspour $^{1}$ (D) . Nasser Mehrshad ${ }^{1}$ (D) . Seyyed Mohammad Razavi ${ }^{1}$ (D)
}

Received: 7 September 2019 / Accepted: 9 January 2020 / Published online: 18 January 2020

(c) Springer Nature Switzerland AG 2020

\begin{abstract}
Removing the contribution of dispensable mental activities dispersed across the electroencephalogram (EEG) signal improves the interpretability and efficiency of the intended neuronal responses to control a brain computer interface $(\mathrm{BCl})$. Performing motor imagery tasks causes proper formation of special patterns at a specific timeframe of the EEG signal. The accurate selection of this optimal informative timeframe has not yet been investigated. Previous studies have considered an identic portion of data for all individuals, while neglecting that the duration and delay takes for the motor imagery brain activities to be well reflected in EEG signals vary between individuals. We propose an intelligent hybrid genetic algorithm — support vector machine (SVM) method to select the most stimulated timeframe of interest. The method also selects the most distinctive subset of features (through a comprehensive fused set of temporal, spectral and wavelet inspected information) while simultaneously optimize the SVM classifier's parameters. Evaluation results show that not only the most stimulated timeframe has a short duration but also occurs after a specific delay: that they are different between individuals. Using this optimal timeframe, the classification accuracy increased up to 92.14\% for Graz 2003 and $89.00 \%, 84.81 \%$ and $85.00 \%$ for O3, S4 and X11 subjects of Graz 2005 database respectively. These results that were obtained despite the use of a small set of features confirm that this intelligent method can be effective in increasing the computational speed while decreasing the computational complexity which provides the potential capability of including in real time $\mathrm{BCl}$ systems.
\end{abstract}

Keywords Brain computer interface $\cdot$ Motor imagery · Timeframe selection · Genetic algorithm · Support vector machine

\section{Introduction}

Brain computer interface $(\mathrm{BCl})$ system enables the users to interact with their environment by intent alone without any intervention of nerves or muscles. Therefore, $\mathrm{BCls}$ considered as potential tools to be used for human to human interfacing [1], neural prosthetics [2], neural gaming [3], exoskeleton control [4], mobile and guided robotics [5], biometrics [6], and Intelligent Transportation [7].

Most of current $\mathrm{BCls}$ use electroencephalogram (EEG) to record brain neural activity, as it benefits from the advantages of non-invasiveness, high temporal resolution, low cost and portability [8-12]. In EEG-based BCls, control signals are obtained by different strategies due to the presence or absence of external stimulus to evoke user intention [13]. Self-modulation of sensory-motor rhythms through motor imagination (MI) of different parts of the body seems to be the most popular paradigm since it can be operated only by the user's will, independently of any external stimulation [14]. The imagination of right or left limbs movement, changing the neuronal activity in contralateral hemisphere of the brain motor cortex leads to

Nasser Mehrshad, nmehrshad@birjand.ac.ir; Hamidreza Abbaspour, hrabbaspour@birjand.ac.ir; Seyyed Mohammad Razavi, smrazavi@birjand.ac.ir|'Department of Electronics, Faculty of Electrical and Computer Engineering, University of Birjand, Birjand, Iran. 
physiological phenomenon known as event-related DE synchronization (ERD) and event-related synchronization (ERS) which are reflected respectively as decreasing and increasing power within either alpha and beta bands in EEG signal [15]. The distinctive pattern of these variations in the signal can be recognized by advanced signal processing and machine learning algorithms to reveal which kind of motion the user has imagined [14].

When dealing with $\mathrm{Ml}$ evoked cerebral signals, one of the main challenging issues is the very large contribution of irrelevant neuronal activities that are not exactly related to the performed imagination task. This misleading information in the signal must be detected and removed prior to further EEG processing as it can reduce interpretability and efficiency of the intended brain neuronal responses in controlling $\mathrm{BCl}$ systems.

Despite many researches have been done on the development of $\mathrm{MI}$ based $\mathrm{BCl}$ systems [9], relatively few strategies have been proposed to isolate additional information especially irrelevant mental activates within the signal. Some researchers have focused on extracting features from entire interval of recorded data and then reduce the features vector dimensions [16-20]. However it is not reasonable to initially consider additional data dispersed over the entire interval of recorded data and then force feature reduction algorithm to remove them. In other words, the task of removing additional information should not be left just for the feature reduction algorithm. Using entire interval of recorded data will increase computational complexity while reducing classification accuracy. In some other studies [21-26], features were extracted from different timeframes obtained by overlapped windowing along the signal and then integrated so that a feature reduction algorithm was applied on the collection of features. This method has also the aforementioned problem of additional data and irrational computation. Because it's possible that feature reduction algorithm selects features from the areas that are not within the interested timeframe of $\mathrm{MI}$ neuronal activities. It should be noted that irrelevant data should not be considered in early stages of the algorithm (feature extraction) so that they do not need to be discarded or be removed hardly later. Therefor the researches [27-32] considered only a particular portion of data, identically for all participants in the experiment: a few seconds after beginning of the imagery task. Indeed, the time takes for MI brain activities to be reflected in EEG signal (delay) and the time takes for it to be completed (duration) varies between individuals. So, the method of using a predefined fixed timeframe reduces the $\mathrm{BCl}$ efficiency since some irrelevant information may be considered while the relevant mental activities may be neglected. Also analytical methods of timeframe selection [33] cannot be good candidates to find the most important information. These methods, in addition to have major shortcomings of high complexity and data dependency, investigate the effects of timeframes on some of statistical criterion instead of the classification accuracy hence they may have little effect on improving the classification results. Furthermore the generalizability of such methods for similar motor imagery tasks is low.

As a given thought can be generated in the brain within a very short timeframe [34], the brain seems to respond quickly to the Ml of various body limbs so that its effect is reflected in a short timeframe in the brain signal. In other words, MI based brain activities are reflected in EEG signals in short timeframe and with specific delay after performing the imagination task so that both of these parameters are different between subjects. Hence, before signal processing, the precise timeframe in which relevant neuronal excitation has been reflected, must be selected and other unnecessary signal segments that do not contain proper brain $\mathrm{Ml}$ activities must be removed. Assuming that each motor imagery task creates ERD/ERS patterns at a specific timeframe of the brain signal, appropriate features describing the pattern at a specified timeframe should be extracted. Obviously, all extracted features from the selected timeframe are not of equal importance in $\mathrm{MI}$ classification tasks. Also, the most important features vary from subject to subject. Therefore an optimal set of the most distinctive features should be selected for each subject. As the search space for such a problem is extensive, a systematic data mining method [35] doesn't seem to be a comprehensive solution. Indeed, an intelligent optimization technique should be provided so that it can automatically find both the optimum timeframe across the EEG signal as well as the optimal set of features within the timeframe.

The evolutionary algorithms employ computational intelligence to find the most optimal solution among a large number of feasible candidates in an extensive search space, while this is very complex for traditional algorithms $[36,37]$. Intelligent optimization techniques concurrently search among a set of feasible solutions to find optimal solutions, while they are not prone to get stuck at the local minimum and do not need to provide information about the solutions $[20,38]$.

In this research, we developed the application of an intelligent evolutionary method to improve the performance of $\mathrm{BCls}$ by detecting and utilizing $\mathrm{MI}$ based neuronal activities in EEG signal and ignoring redundant contributions. Specifically a combination of genetic algorithm (GA) and support vector machine (SVM) classifier has been applied to determine the optimal timeframe (on which MI brain activities are properly reflected) and select the most distinctive subset of features extracted within the optimal timeframe. Good performance of the GA when used with 
SVM classifier for dealing with the EEG signals has been proven in several researches [36, 39-41]. This method is inspired by a wrapper selection process, which is a popular algorithm in the machine learning researches [42].

\section{Methods}

The current investigation involved an intelligent data mining based method of Identifying the most important information in the EEG signals to improve the efficiency of $\mathrm{BCl}$ systems.

Experiment Signals were recorded from $\mathrm{C} 3$ and $\mathrm{C} 4$ areas of the brain motor cortex which have been frequently used to interpret user intentions during movement imagination of the user's right and left hands. A third order Butterworth band pass $(8-30 \mathrm{~Hz})$ filter was applied on the signals as the activity of excited neurons is mainly reflected in this frequency range. The hybrid GA-SVM algorithm was performed to find the most stimulated EEG signal timeframe containing relevant $\mathrm{MI}$ patterns so that the appropriate characterizing features of only the enclosed patterns in the timeframe could be extracted. The combination was achieved when SVM oriented GA to explore the search space for the most optimal solution by giving value to each of the feasible solutions (chromosomes). The features set, which were obtained from signal representation in temporal, spectral and wavelet domains, was inspected by the hybrid GA-SVM method to select the most distinctive subset of features that is more effective in discriminating right and left hand movement imaginations. Performance was checked over tenfolds cross validation results and the model parameters were automatically adjusted during the wrapper process for the best generalization to be obtained.

As mentioned above, the main steps of the proposed method were conducted using a GA wrapped with an SVM classifier. The flowchart of the algorithm is presented in Fig. 1. More details are described below.

\subsection{Experimental data}

Two public datasets of Graz 2003 (dataset III) [43] and Graz 2005 (dataset IIIB) [44] that have been prepared by Graz University of Technology were used to investigate the effectiveness of the proposed method.
Fig. 1 Flowchart of the proposed method
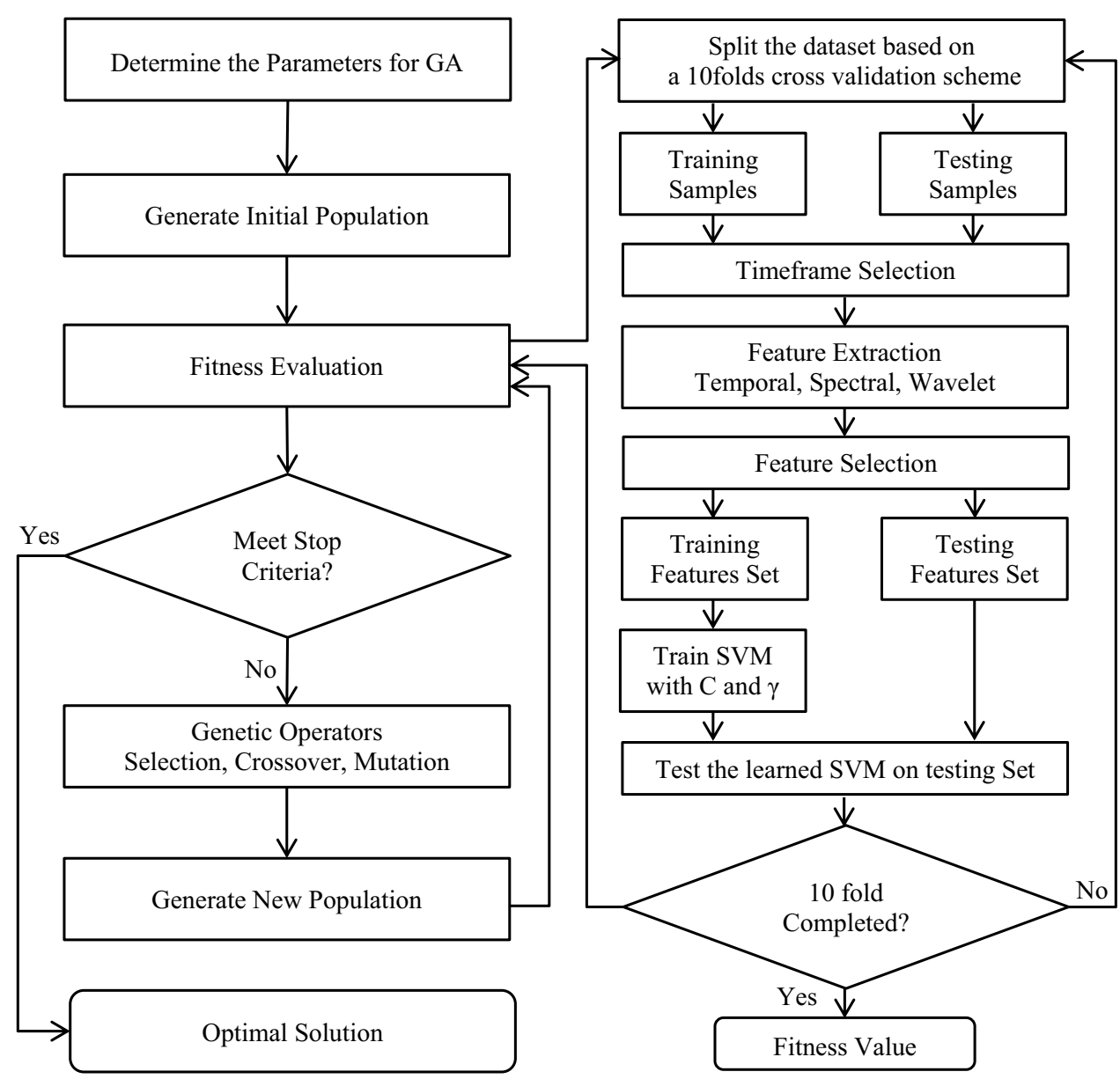

SN Applied Sciences 
Dataset III signals were recorded during a feedback process from C3, Cz and C4 brains areas of a normal 25 years old female. The experiment consisted of 7 runs all conducted in a same day with 40 trials of $9 \mathrm{~s}$ in each that were sampled with a rate of $128 \mathrm{~Hz}$. Nothing happens in the first $2 \mathrm{~s}$ until at $\mathrm{t}=2 \mathrm{~s}$, an acoustic alarm specifies the beginning of the experiment. Then at $\mathrm{t}=3 \mathrm{~s}$ a visual cue to the right and left displayed and the subject was asked to move a feedback bar in the same direction, by doing movement imagination of her left and right hands.

Dataset IIIB signals were recorded during a cued motor imagery task with online feedback from $\mathrm{C} 3$ and $\mathrm{C} 4$ brains areas of three subjects $(\mathrm{X} 11, \mathrm{~S} 4, \mathrm{O} 3)$. Experiments were conducted in three sessions each of which includes 4-9 runs. The duration of each trail was $8 \mathrm{~s}$ and the records were sampled with a rate of $125 \mathrm{~Hz}$. Nothing happens in the first $2 \mathrm{~s}$ until at $\mathrm{t}=2 \mathrm{~s}$, an acoustic alarm specifies the beginning of the experiment. Then at $t=3 \mathrm{~s}$ a cue to the right or left (virtual reality for $\mathrm{O} 3$ or basket for $\mathrm{S} 4$ and $\mathrm{X} 11$ ) was displayed, and the subject was asked to move a feedback bar in the same direction, by doing movement imagination of left and right hands.

The experimental paradigm in the datasets is shown in Fig. 2.

Details describing the datasets are displayed in Table 1.

\subsection{Timeframe selection}

In order to accurately identify the user intentions, features must be taken from the exact timeframe of acquired brain signals which reflects relevant brain activities. Detection of this optimal informative timeframe was performed by the hybrid GA-SVM algorithm which exploited computational intelligence to find two characteristic parameters of the timeframe: starting point and the duration of the timeframe. In GA, feasible solutions or search agents are referred as chromosomes each of which composed of several gens that are parametric components of a solution. Here we assigned the first two genes of a chromosome for the timeframe characteristic parameters (as illustrated in Fig. 3). The applied settings for GA were included: generation size 500 , population size 100 , stall generation limit 50 , stochastic uniform selection, adaptive feasible mutation and Intermediate crossover function. The best member of

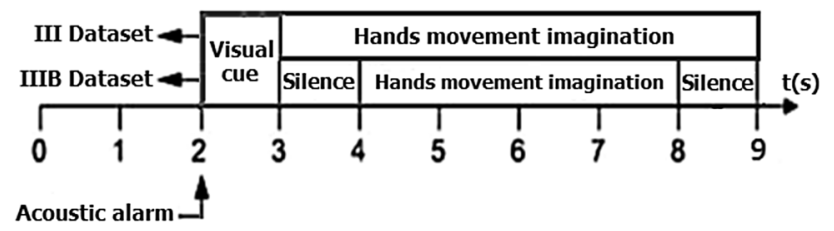

Fig. 2 Experimental paradigm of the datasets
Table 1 Describing details of the datasets

\begin{tabular}{lllll}
\hline Database & III & \multicolumn{2}{l}{ IIIB } & \\
\cline { 3 - 5 } & & $\mathrm{O}_{3}$ & $\mathrm{~S}_{4}$ & $\mathrm{X}_{11}$ \\
\hline Parameter & & & & \\
Number of trails & 280 & 300 & 520 & 520 \\
Feedback type & $\mathrm{Bar}$ & $\mathrm{Bar}$ & Basket & Basket \\
Number of classes & 2 & 2 & 2 & 2 \\
Recorded signal duration (s) & 9 & 8 & 7 & 7 \\
Feedback presentation (s) & $3-9$ & $4-8$ & $4-7$ & $4-7$
\end{tabular}

the final generation was selected as the solution of the problem.

\subsection{Feature extraction}

A total of 30 features were extracted from the considered timeframe of $c 3$ and $c 4$ EEG channels so that each set of 10 features, represents the signals characteristics in one of temporal, spectral and wavelet domains. Cz channel of III database was ignored since it has been shown to be independent of motor imagery brain activities [23].

Temporal features due to their low computational complexity are good options for use in $\mathrm{BCl}$ systems [31]. Computed temporal features included mean absolute deviation, skewness, kurtosis, Hjorth parameters, Shannon entropy, waveform length, number of local peaks and number of zero crossings. Mean absolute deviation involves the total variability of the EEG signals [45]. Skewness and kurtosis are measures of asymmetry and tailedness of the amplitude distribution of the EEG signal respectively [46]. Hjorth parameters that include activity, mobility and complexity are commonly used indicators of EEG signal's statistical properties in time domain. That is activity describes the appearance of power spectrum in frequency domain, mobility represents the proportion of standard deviation of the power spectrum and complexity indicates the similarity degree of the signal to a pure sin wave [47]. The irregularity degree of the patterns in the signal is characterized by information or Shannon entropy [48]. A measure of waveform complexity can be provided by waveform length which is defined as the cumulative length of the signal within the timeframe [31, 49]. Number of local peaks and zero crossings are simple measures

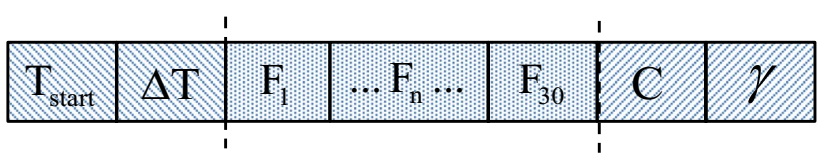

Fig. 3 The chromosome consists of 3 sets: timeframe characteristics, feature subset and SVM parameters 
related with the frequency information of the signal that are defined in time domain [49].

Spectral features are one of the most commonly used feature extraction techniques in sensorimotor based $\mathrm{BCl}$ systems $[27,50]$. We calculated spectral features from the power spectral density (PSD) of the timeframe (via Welch period gram) that provide signal power in our interested frequency bands (alpha and beta) in which ERD/ERS occurred. Spectral features included total power (also called zero spectral moment), first and second spectral moments, mean frequency and power spectrum deformation, were estimated independently across alpha and beta bandwidths. These features were considered, as they are important analytical statistics to summarize signal power spectrum properties [51, 52].

Wavelet transform benefits the advantages of converting the signal into frequency domain, while simultaneously keeping the rich characteristics of the original signal in time domain. Wavelet features were extracted by applying a series of widely used statistics in neurological activity analysis (sub band energy, root mean square, mean absolute value, integrated EEG, average of the coefficients) $[46,49,53,54]$ to the discrete wavelet transform (DWT) coefficients of the first and second decomposition levels, occupying beta and alpha frequency bands, respectively.

In multi sessions $\mathrm{BCl}$ recording, EEG data have inherent non stationary characteristics. Therefore, extracted features will be un-stable from training data to testing data. In order to eliminate the possibility of high variance features dominating the classification training and testing phases, a normalization method was used after extracting features by rescaling the features to have zero mean and unity standard deviation.

\subsection{Feature selection}

Selecting the appropriate features, directly affects the $\mathrm{BCl}$ system performance. All the extracted features do not have the ability to distinguish brain activities so that additional and inappropriate features can mislead the classifier to make wrong decisions, thus decreasing the overall $\mathrm{BCI}$ system performance. By preserving the most distinctive features and removing the redundant ones, the curse of dimensionality problem and over-fitting were avoided, so that in addition to reducing the computational cost and complexity, the classifier can have better generalizability.

The hybrid GA-SVM algorithm was applied to select the optimal subset of features. Genes from number 3-32 (the same size as the features vector) assigned for feature selection (see Fig. 3) and designed in such a way to have binary content where 1 and 0 corresponded to selecting and discarding the corresponding feature, respectively.

\subsection{Classification}

The classification was performed by SVM classifier which is a common, state of the art, and efficient method for $\mathrm{BCl}$ applications [31, 55, 56]. Using the kernel function, SVM classifier can create nonlinear hyper plates to discriminate the data of each class by maximizing the margin across the classes and minimizing misclassified samples. We considered Gaussian radial basis function (RBF) as the kernel function, since it was suggested as the most suitable choice $[27,57]$. Furthermore it has the lowest number of hyper parameters (only $C$ and $\gamma$ ) compared to other kernel functions whose configuration adjustment is less expensive. Penalty factor or $\mathrm{C}$ is a regularization parameter to tune the misclassification rate of the training data. Kernel factor or $\gamma$ affects the shape of the Gaussian RBF function. For improving the classifier capability to achieve satisfactorily results, we need to find the optimal values of these hyper parameters. Fine tuning these parameters will optimize the compromise between bias and variance which are related with the accuracy and complexity of the model and affect the generalizability and efficiency of the model.

The hybrid GA-SVM approach, as a powerful intelligent strategy was used to select the optimal values of these parameters, instead of the traditional Grid search algorithm [58] which was time-consuming and may not provide good results. The penultimate and the last gens of the chromosomes where assigned to $C$ and $\gamma$ (see Fig. 3) so that they were set to take numbers in the range of $\left[2^{-5}, 2^{15}\right]$ and $\left[2^{-15}, 2^{3}\right]$ respectively. Such intervals were considered to limit the search boundaries of GA agents as they were recommended by several investigations $[59,60]$.

\section{Results}

The main steps of our proposed method were conducted using a GA wrapped with an SVM classifier. GA in each generation calls SVM classifier to evaluate the fitness of a set of variables (chromosomes or feasible solutions) on the database. The calculated fitness by the SVM classifier is then fed back to the GA to generate a new set of variables that better estimate the optimal (global) response of the problem.

When the structure of the chromosome was configured by GA (as shown in Fig. 3), each set of its components was referred to its duties: selecting timeframe (Sect. 2.2), selecting features (Sect. 2.4) and providing the classifier parameters (Sect. 2.5) while the two formers were performed simultaneously for both $\mathrm{c} 3$ and $\mathrm{c} 4$ channels. In other words, based on the components of each chromosome (gens), the timeframe is selected from the signals (by the first two genes) to extract the relevant features. Then the 
Table 2 Optimal values calculated for the databases

\begin{tabular}{lllll}
\hline Database & III & IIIB & & \\
\cline { 3 - 5 } & & $\mathrm{O}_{3}$ & $\mathrm{~S}_{4}$ & $\mathrm{X}_{11}$ \\
\hline Parameter & & & & \\
Timeframe delay (s) & 0.80 & 0.02 & 2.06 & 0.97 \\
Timeframe duration (s) & 0.96 & 0.85 & 0.79 & 1 \\
Total no. of selected features & 5 & 8 & 5 & 9 \\
C & 12.31 & 3.86 & 15.09 & 3.10 \\
Gamma & 3.95 & 0.84 & 5.69 & 4.20 \\
\hline
\end{tabular}

Table 3 Number of selected features from different extracted feature groups

\begin{tabular}{llll}
\hline Features & $\begin{array}{l}\text { Temporal } \\
\text { (out of 10) }\end{array}$ & $\begin{array}{l}\text { Wavelet } \\
\text { (out of 10) }\end{array}$ & $\begin{array}{l}\text { Spectral } \\
\text { (out of 10) }\end{array}$ \\
\hline $\begin{array}{lll}\text { Database } \\
\text { III }\end{array}$ & 2 & 2 & 1 \\
O3 & 1 & 2 & 5 \\
S4 & 4 & 0 & 1 \\
X11 & 2 & 3 & 4 \\
\hline
\end{tabular}

most distinguishing features among the extracted ones (by gene number 3-32) as well as the classifier parameters (by the penultimate and the last gens) are selected to perform the classification task and fitness assignment to the chromosome.

Classification accuracy was considered to assess the fitness (i.e. quality) of each chromosome; hence in our GASVM model the search directions toward the best classification accuracy in final iteration were determined based on the feedback from SVM to GA. To prevent over-fitting and improve the method's generalizability, the classification accuracy corresponding to each chromosome was estimated based on a tenfold cross validation scheme over the test data. The results from tenfolds were then averaged to produce an estimation of the chromosome's fitness. The fittest chromosome in the last generation determines the optimal solution of the problem. The obtained solutions are reported in Table 2.

Table 3 shows in detail the number of selected features from different extracted feature groups. These values were obtained by considering the optimal solution of the problem.

Selected features from both c3 and c4 channels of III dataset for left and right hand movement imaginations were demonstrated by box plots in Fig. 4. The box plots indicate low within class variance and good between class
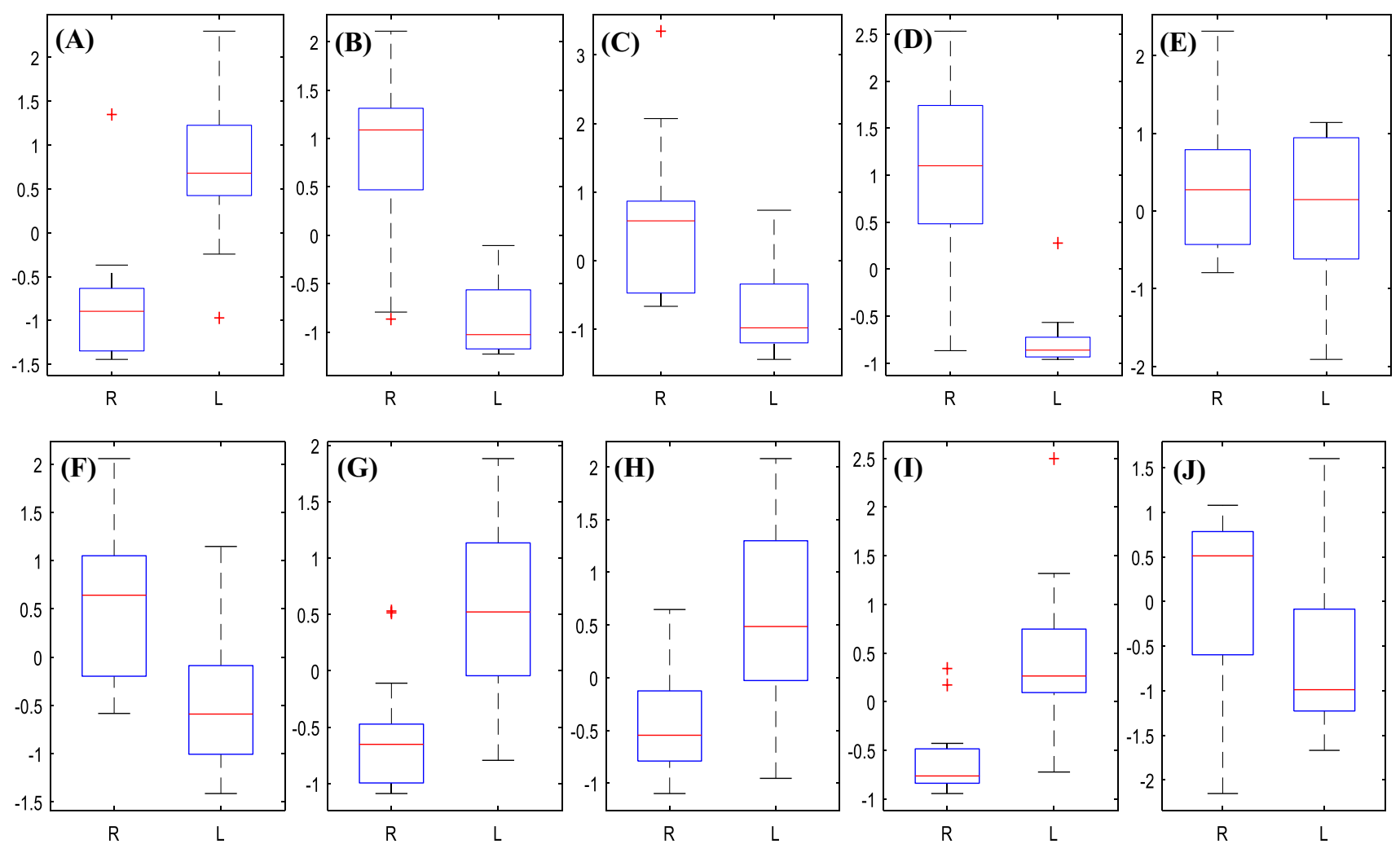

Fig. 4 Boxplots of selected features for right and left hand movement imaginations of c3 (a-e) and c4 (f-j) channels in dataset III 
distances of the selected features in the feature vector space. This means that the selected features have distinctive values for the two specific movement imaginations and are of high quality for use in classifying and identifying right and left hand movement imagination tasks.

The normalized distribution of selection probability for each signal sample is shown in Fig. 5. The height of each bar is equivalent to the times the sample corresponding to that bar was selected, divided by the times that entire signal samples were selected by the algorithm.

Figure 6 depicts the selection probability distribution of each extracted feature. The height of each bar is equivalent to the total times that each feature was selected divided by total times that entire extracted features were selected.

The accuracy of the method as an important criterion for assessing the efficiency of the proposed model was calculated on the fittest chromosome of the last generation. In addition, the classification performance was evaluated in detail by other statistical criteria, such as sensitivity and specificity in the form of a confusion matrix reported in Table 4.

The performance comparison of the proposed method with existing studies which have been tested on Graz 2003 and Graz 2005 databases, are summarized in Tables 5 and 6.

\section{Discussion}

In the current investigation we propose a novel intelligent method to improve the classification accuracy of right and left hands movement imagination by identifying and separating corresponding brain activity from EEG signals acquired during a BCl paradigm. Getting rid of additional information dispersed across the EEG signal by removing the contribution of irrelevant
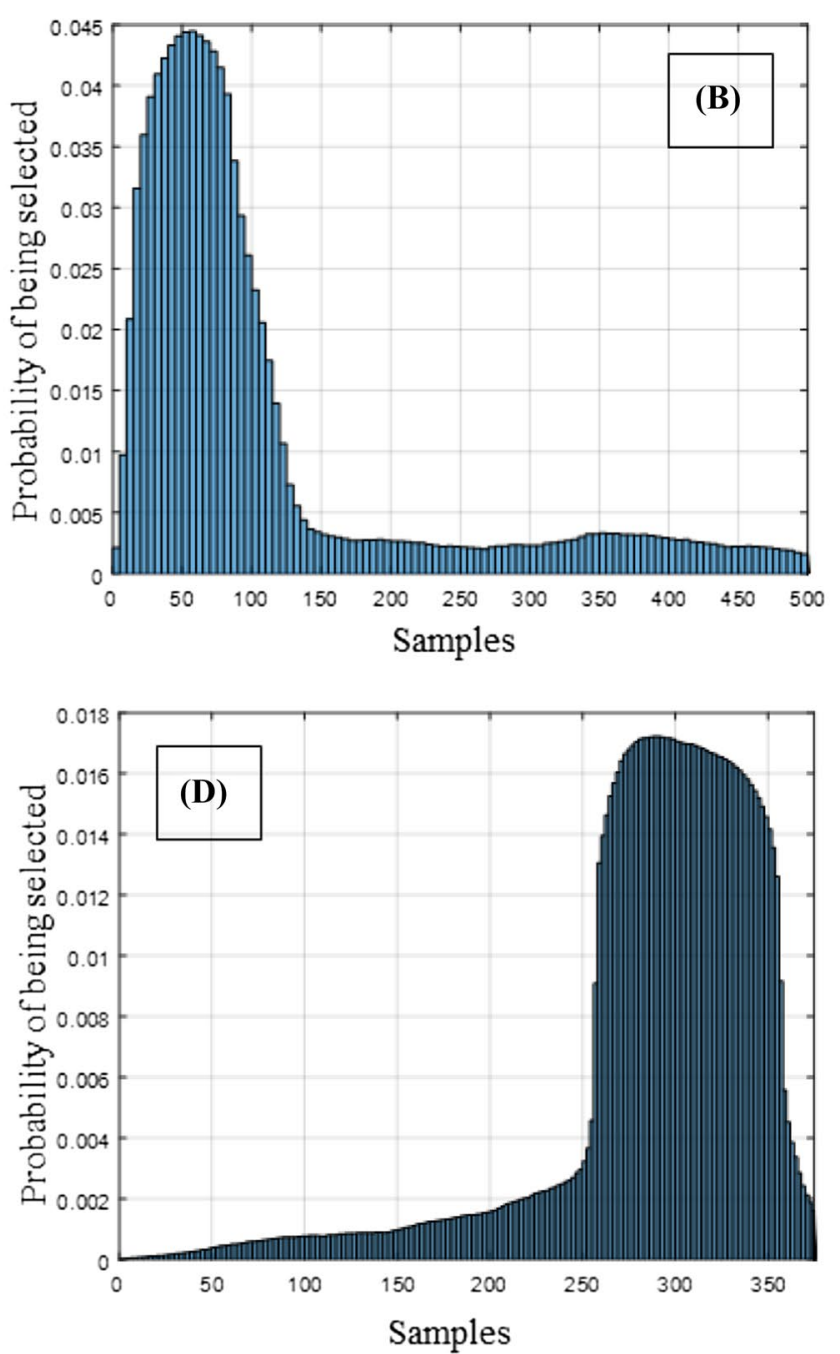

Fig. 5 Signal samples selection probability for III (a), O3 (b), X11 (c) and S4 (d) datasets 

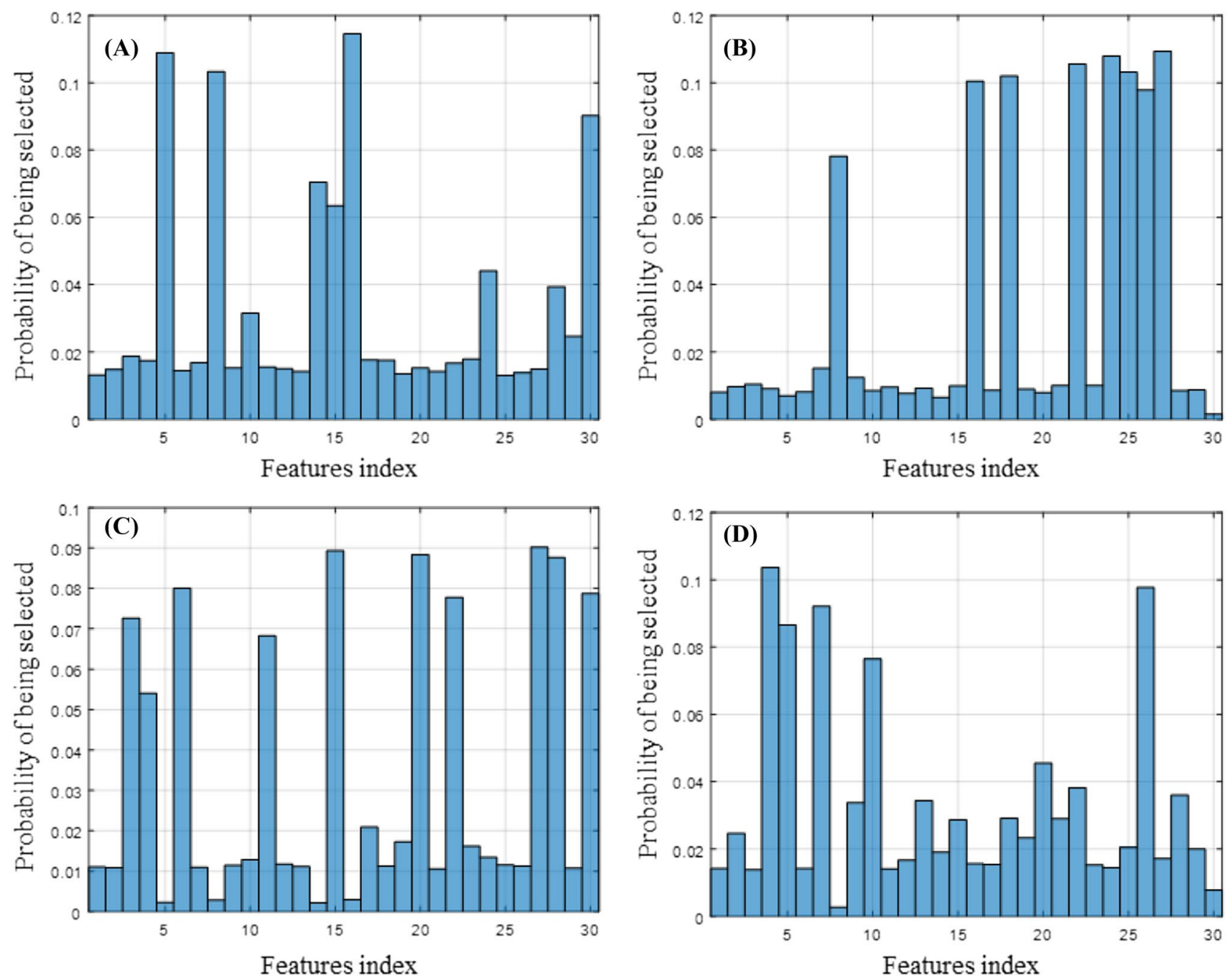

Fig. 6 Extracted features selection probability for III (a), O3 (b), X11 (c) and S4 (d) datasets

Table 4 Confusion matrix

\begin{tabular}{|c|c|c|c|c|c|c|}
\hline \multirow[t]{2}{*}{ Database } & \multirow[t]{2}{*}{ Output } & \multicolumn{2}{|c|}{ Target } & \multirow[t]{2}{*}{ Sensitivity (\%) } & \multirow[t]{2}{*}{ Specificity (\%) } & \multirow[t]{2}{*}{ Accuracy (\%) } \\
\hline & & Left & Right & & & \\
\hline III & $\begin{array}{l}\text { Left } \\
\text { Right }\end{array}$ & $\begin{array}{l}13.10 \\
0.90\end{array}$ & $\begin{array}{l}1.30 \\
12.70\end{array}$ & 90.97 & 93.38 & 92.14 \\
\hline S4 & $\begin{array}{l}\text { Left } \\
\text { Right }\end{array}$ & $\begin{array}{l}21.80 \\
4.20\end{array}$ & $\begin{array}{l}3.70 \\
22.30\end{array}$ & 85.49 & 84.15 & 84.81 \\
\hline $\mathrm{O} 3$ & $\begin{array}{l}\text { Left } \\
\text { Right }\end{array}$ & $\begin{array}{l}12.90 \\
2.10\end{array}$ & $\begin{array}{l}1.20 \\
13.80\end{array}$ & 91.49 & 86.79 & 89.00 \\
\hline $\mathrm{X} 11$ & $\begin{array}{l}\text { Left } \\
\text { Right }\end{array}$ & $\begin{array}{l}22.30 \\
3.70\end{array}$ & $\begin{array}{l}4.10 \\
21.90\end{array}$ & 84.47 & 85.55 & 85.00 \\
\hline
\end{tabular}

and dispensable mental activities from the following phases of signal processing procedure (e.g. feature extraction) improves the interpretability and efficiency of the intended mental responses to control the $\mathrm{BCl}$ systems. Prior works have confirmed the impact of isolating redundant contributions in the EEG signal to benefit from the proper relevant information by using either fixed [27-32] or sets of overlapped [21-26] timeframes selected along the signal. As stated in the introduction section, these methods cannot be the right strategies. MI based brain activities are well reflected in EEG signals in a short timeframe and with a short delay after 
Table 5 Accuracy comparison of the proposed method with existing methods for Graz 2003

\begin{tabular}{llll}
\hline Study & Feature extraction technique & $\begin{array}{l}\text { Classification tech- } \\
\text { nique }\end{array}$ & $\begin{array}{l}\text { Correct clas- } \\
\text { sification rate }\end{array}$ \\
\hline Xu et al. & DWT & FSVM & 80.71 \\
Biag et al. & WT & SVM & 88.57 \\
Wei et al. & EMD/IMF & FCM & 78.00 \\
Zhou et al. & AR/PSD/bi-spectrum & NN & 90.00 \\
Proposed method & Temporal/spectral/DWT & SVM & 92.14 \\
\hline
\end{tabular}

DWT discrete wavelet transform, FSVM fuzzy support vector machine, WT wavelet transform, SVM support vector machine, $E M D$ empirical mode decomposition, IMF intrinsic mode function, FCM fuzzy $c$ means, $A R$ autoregressive, PSD power spectral density, NN neural network, PCA principal components analysis, ERNN Elman recurrent neural network

\begin{tabular}{llllll}
\hline Study & Feature extraction technique & Classification & \multicolumn{2}{l}{ Correct classification rate } \\
\cline { 5 - 6 } & & technique & $\mathrm{O}_{3}$ & $\mathrm{~S}_{4}$ & $\mathrm{X}_{11}$ \\
\hline Rod et al. & PSD/Hjorth/AR/CWT & LOO & 68.95 & 77.78 & 67.18 \\
Fang et al. & AFAPS/ARPS & LDA & 87.50 & 75.10 & 84.54 \\
Chen et al. & AFAPS & LDA & 85.53 & 76.95 & 73.18 \\
Bash et al. & Morlet & MLP & 83.65 & 82.22 & 76.67 \\
Proposed method & Temporal/spectral/DWT & SVM & 89.00 & 84.81 & 85.00 \\
\hline
\end{tabular}

PSD power spectral density, AR autoregressive, CWT continuous wavelet transform, $L O O$ leave one out, AFAPS amplitude frequency analysis in phase space, ARPS autoregressive modeling in phase space, $L D A$ linear discriminant analysis, MLP multi layer perceptron, DWT discrete wavelet transform, $P C A$ principal components analysis, ERNN Elman recurrent neural network
Table 6 Accuracy comparison of the proposed method with existing methods for Graz 2005 features) while simultaneously optimize the classifier's parameters.

The performance of the proposed method was evaluated using two public $\mathrm{BCl}$ competition databases. To ensure the generalizability of the method, the classification results were calculated during a cross validation scheme over the test data. Remarkable results were obtained for classification of right and left hand movement imaginations by using the optimal values of the problem unknown components: timeframe, features and SVM parameters. The results reported in Table 2 indicate that the proper MI patterns are not dispersed across the signal so that the optimal timeframe has a short duration (less than a second) and occurs after a specific delay: both the delay and the duration are different between individuals. Figure 5 clearly depicts these findings. As observed in Fig. 5 a specific timeframe, which is actually the same optimal timeframe contains MI patterns, has been frequently investigated by GA. This is because SVM classifier has forwarded better responses to GA in this timeframe's neighborhood. Since farther signal portions from this neighborhood didn't contain rich MI information, they have led to poor classification accuracy and have been abandoned from the GA's search space at initial iterations. Figure 6 depicts that $5,8,9$ and 5 features (each of which, out of 30 
extracted features) were selected more than the rest, considering different datasets. This has happened due to these features special ability in distinguishing the movement imagination of left and right hands. The same features were selected by the algorithm as the final optimal subset of features. By considering whole selected features for all databases, the spectral features were more distinguishable because the most selected features were from the spectral features group (Table 3). The high quality of the selected features is clearly seen in Fig. 4 (briefly, only for database III) by considering the low within class variance and good between class distances in the feature vector space. The confusion matrix given in Table 4 includes high values in its diagonal elements, which confirms the ability of the classifier in correctly identifying MI brain activities. Furthermore, the results reported in Table 4 show that the proposed method present's high values of accuracy, sensitivity and specificity for the classification task. Comparing classification accuracy of the proposed method with existing methods reported in Tables 5 and 6 indicate that our new proposed method, despite using lower number of features, provides better identification results.

\section{Conclusion}

An innovative algorithm is proposed to identify Ml activities in $\mathrm{BCl}$ systems. Promising results are obtained, indicating that our novel algorithm is able to effectively select the optimal sensorimotor reaction timeframe across the EEG signal in a $\mathrm{MI}$ based $\mathrm{BCl}$ paradigm.

The superior performance of the proposed method was achieved by using only a few features, resulting in high information transfer rate, less computational complexity and requiring less storage space. It's worth mentioning that GA is simply parallelizable to accelerate the execution speed. However, GA is performed only in offline mode to find optimal variables so that in online mode those variables are used without needing for the GA to be executed. Therefore, rather than increasing the computational time, GA guides to achieve the best results quickly by providing the optimal parameters. Another important advantage of the proposed method is the significant reduction in the duration of the brain signal recording time, which is necessary in a $\mathrm{BCl}$ system for translating the user's intentions into commands and creating the control signal. Although the time latency needed to generate the control signal in other methods was at least equal to the total duration of the recorded signal, it will be much shorter in the proposed method, since the optimal timeframe calculated in all datasets has a short duration of less than a second.

While these advantages reveal the effectiveness of the proposed method both for offline and online $\mathrm{BCl}$ applications, testing it in an online environment can be a potential perspective for the future work. Furthermore, we only considered the data recorded from healthy subjects: developing the study to patients with various disabilities can be a next major step to be addressed.

\section{Compliance with ethical standards}

Conflict of interest The authors declared that they have no conflict of interest.

\section{References}

1. Kapur A, Kapur S, Maes P (2018) AlterEgo: a personalized wearable silent speech interface. In: 23rd International conference on intelligent user interfaces. ACM, pp 43-53

2. Malik AN, Iqbal J, Tiwana MI (2016) Temporal based EEG signals classification for talocrural and knee joint movements using emotive head set. J Biomed Eng Med Imaging 2:69

3. Marshall D, Coyle D, Wilson S, Callaghan M (2013) Games, gameplay, and BCl: the state of the art. IEEE Trans Comput Intell AI Games 5:82-99

4. Semprini M, Laffranchi M, Sanguineti V et al (2018) Technological approaches for neurorehabilitation: from robotic devices to brain stimulation and beyond. Front Neurol 9:212

5. Nurseitov D, Serekov A, Shintemirov A, Abibullaev B (2017) Design and evaluation of a P300-ERP based $\mathrm{BCl}$ system for realtime control of a mobile robot. In: 5th International winter conference on brain-computer interface (BCI). IEEE, pp 115-120

6. Saulynas S, Lechner C, Kuber R (2018) Towards the use of braincomputer interface and gestural technologies as a potential alternative to PIN authentication. Int J Hum Comput Interact 34:433-444

7. Fan X, Bi L, Teng T et al (2015) A brain-computer interface-based vehicle destination selection system using P300 and SSVEP signals. IEEE Trans Intell Transp Syst 16:274-283

8. Mihajlović V, Grundlehner B, Vullers R, Penders J (2015) Wearable, wireless EEG solutions in daily life applications: what are we missing? IEEE J Biomed Health Inform 19:6-21

9. McFarland DJ, Wolpaw JR (2017) EEG-based brain-computer interfaces. Curr Opin Biomed Eng 4:194-200

10. Barua S, Ahmed MU, Ahlstrom C et al (2017) Automated EEG artifact handling with application in driver monitoring. IEEE J Biomed Health Inform 22:1350-1361

11. Somers B, Francart T, Bertrand A (2018) A generic EEG artifact removal algorithm based on the multi-channel Wiener filter. J Neural Eng 15:036007

12. Padfield N, Zabalza J, Zhao H et al (2019) EEG-based braincomputer interfaces using motor-imagery: techniques and challenges. Sensors 19:1423

13. Abdulkader SN, Atia A, Mostafa M-SM (2015) Brain computer interfacing: applications and challenges. Egypt Inform J $16: 213-230$

14. Martín-Clemente R, Olias J, Thiyam D et al (2018) Information theoretic approaches for motor-imagery $\mathrm{BCl}$ systems: review and experimental comparison. Entropy 20:7

15. Nicolas-Alonso LF, Gomez-Gil J (2012) Brain computer interfaces: a review. Sensors 12:1211-1279

16. Bashashati H, Ward RK, Birch GE, Bashashati A (2015) Comparing different classifiers in sensory motor brain computer interfaces. PLoS ONE 10:e0129435 
17. Baig MZ, Mehmood Y, Ayaz Y (2016) A BCl system classification technique using median filtering and wavelet transform. In: Kotzab H, Pannek J, Thoben KD (eds) Dynamics in logistics. Springer, Berlin, pp 355-364

18. Wei $Y$, Jun $Y$, Lin $S$, Hong $L$ (2014) Improving classification accuracy using fuzzy method for $\mathrm{BCl}$ signals. Bio-Med Mater Eng 24:2937-2943

19. Bhattacharyya S, Sengupta A, Chakraborti T et al (2014) Automatic feature selection of motor imagery EEG signals using differential evolution and learning automata. Med Biol Eng Comput 52:131-139

20. Hsu W-Y (2014) Improving classification accuracy of motor imagery EEG using genetic feature selection. Clin EEG Neurosci 45:163-168

21. Chen M, Fang Y, Zheng X (2014) Phase space reconstruction for improving the classification of single trial EEG. Biomed Signal Process Control 11:10-16

22. Fang $Y$, Chen $M$, Zheng $X$ (2015) Extracting features from phase space of EEG signals in brain-computer interfaces. Neurocomputing 151:1477-1485

23. Xu Q, Zhou H, Wang Y, Huang J (2009) Fuzzy support vector machine for classification of EEG signals using wavelet-based features. Med Eng Phys 31:858-865

24. Zhou S-M, Gan JQ, Sepulveda F (2008) Classifying mental tasks based on features of higher-order statistics from EEG signals in brain-computer interface. Inf Sci 178:1629-1640

25. Rodríguez-Bermúdez G, García-Laencina PJ, Roca-González J, Roca-Dorda J (2013) Efficient feature selection and linear discrimination of EEG signals. Neurocomputing 115:161-165

26. Yaacoub C, Mhanna G, Rihana S (2017) A genetic-based feature selection approach in the identification of left/right hand motor imagery for a brain-computer interface. Brain Sci 7:12

27. Fouad IA, Hadidi T (2015) Classifying brain-computer interface features based on statistics and density of power spectrum. Int J Biomed Eng Technol 18:1-13

28. Qiu Z, Jin J, Lam H-K et al (2016) Improved SFFS method for channel selection in motor imagery based $\mathrm{BCl}$. Neurocomputing 207:519-527

29. Arvaneh M, Guan C, Ang KK, Quek C (2013) EEG data space adaptation to reduce intersession nonstationarity in brain-computer interface. Neural Comput 25:2146-2171

30. Oikonomou VP, Georgiadis K, Liaros G et al (2017) A comparison study on EEG signal processing techniques using motor imagery EEG data. In: IEEE 30th international symposium on computerbased medical systems (CBMS). IEEE, pp 781-786

31. Tavakolan M, Frehlick Z, Yong X, Menon C (2017) Classifying three imaginary states of the same upper extremity using time-domain features. PLoS ONE 12:e0174161

32. Li M, Zhu W, Liu H, Yang J (2017) Adaptive feature extraction of motor imagery EEG with optimal wavelet packets and SE-isomap. Appl Sci 7:390

33. Zhong M, Lotte F, Girolami M, Lécuyer A (2008) Classifying EEG for brain computer interfaces using Gaussian processes. Pattern Recogn Lett 29:354-359

34. Grinde B, Grinde B (2016) Evolution of consciousness. Springer, Berlin

35. Abbaspour H, Mehrshad N, Razavi SM (2018) An effective braincomputer interface system based on the optimal timeframe selection of brain signals. Int Clin Neurosci J 5:35-42

36. Abbaspour H, Mehrshad N, Razavi SM, Mesin L (2019) Artefacts removal to detect visual evoked potentials in brain computer interface systems. J Biomim Biomater Biomed Eng 41:91-103

37. Nakisa B, Rastgoo MN, Tjondronegoro D, Chandran V (2017) Evolutionary computation algorithms for feature selection of EEGbased emotion recognition using mobile sensors. Expert Syst Appl 93:143-155
38. Rejer I (2015) Genetic algorithms for feature selection for brain computer interface. Int J Pattern Recognit Artif Intell 29:1559008

39. Liang Z, Huang C, Li Y et al (2018) Emergence EEG pattern classification in sevoflurane anesthesia. Physiol Meas 39:45006

40. Yang $\mathrm{H}$, Wu S (2014) EEG classification for $\mathrm{BCl}$ based on CSP and SVM-GA. Appl Mech Mater 459:228-231

41. Shen C-P, Lin J-W, Lin F-S et al (2017) GA-SVM modeling of multiclass seizure detector in epilepsy analysis system using cloud computing. Soft Comput 21:2139-2149

42. Zahedi H, Mehrshad N, Anvari K (2012) Intelligent modelling of oesophageal cancer treatment and its use to determine the dose of chemotherapy drug. J Med Eng Technol 36:261-266

43. A S, Neuper C, Müller GR et al (2002) BCl competition II. http:// www.bbci.de/competition/ii/. Accessed 1 Oct 2016

44. Schlögl A, Graimann B, Pfurtscheller G (2005) BCl competition III. http://www.bbci.de/competition/iii/. Accessed 1 Oct 2016

45. Vigneshwari C,VimalaV, Vignesh SV, Sumithra G (2013) Analysis of finger movements using EEG signal. Int J Emerg Technol Adv Eng 3:583-588

46. Altın C, Er O (2016) Comparison of different time and frequency domain feature extraction methods on elbow gesture's EMG. Eur $J$ Interdiscip Stud 5:35-44

47. Oh S-H, Lee $\mathrm{Y}-\mathrm{R}$, Kim H-N (2014) A novel EEG feature extraction method using Hjorth parameter. Int J Electron Electr Eng 2:106-110

48. Ni Z, Wang L, Meng J et al (2011) EEG signal processing in anesthesia feature extraction of time and frequency parameters. Procedia Environ Sci 8:215-220

49. Tkach $D$, Huang $H$, Kuiken TA (2010) RSetseuardchy of stability of time-domain features for electromyographic pattern recognition. J Neuro Eng Rehabil 5:7-21

50. Bascil MS, Tesneli AY, Temurtas F (2016) Spectral feature extraction of EEG signals and pattern recognition during mental tasks of 2-D cursor movements for $\mathrm{BCl}$ using SVM and ANN. Australas Phys Eng Sci Med 39:665-676

51. Phinyomark A, Phukpattaranont P, Limsakul C (2012) Feature reduction and selection for EMG signal classification. Expert Syst Appl 39:7420-7431

52. Kendell C, Lemaire ED, Losier $Y$ et al (2012) A novel approach to surface electromyography: an exploratory study of electrode-pair selection based on signal characteristics. J Neuroeng Rehabil 9:24

53. Sankar AS, Nair SS, Dharan VS, Sankaran P (2015) Wavelet sub band entropy based feature extraction method for $\mathrm{BCl}$. Procedia Comput Sci 46:1476-1482

54. Alomari MH, Awada EA, Samaha A, Alkamha K (2014) Waveletbased feature extraction for the analysis of EEG signals associated with imagined fists and feet movements. Comput Inf Sci 7:17

55. Selim S, Tantawi MM, Shedeed HA, Badr A (2018) A CSP \AMBA-SVM approach for motor imagery $\mathrm{BCl}$ system. IEEE Access 6:49192-49208

56. Santamaria L, James C (2018) Using brain connectivity metrics from synchrostates to perform motor imagery classification in EEG-based BCl systems. Healthc Technol Lett 5:88-93

57. Kim C, Sun J, Liu D et al (2018) An effective feature extraction method by power spectral density of EEG signal for 2-class motor imagery-based $\mathrm{BCl}$. Med Biol Eng Comput 1-14

58. Hsu CW, Chang CC, Lin CJ (2016) A practical guide to support veCtor classification. National Taiwan University, Taipei

59. Liao P, Zhang X, Li K et al (2015) Parameter optimization for support vector machine based on nested genetic algorithms. J Autom Control Eng 3:507-511

60. Phan AV, Le Nguyen M, Bui LT (2017) Feature weighting and SVM parameters optimization based on genetic algorithms for classification problems. Appl Intell 46:455-469

Publisher's Note Springer Nature remains neutral with regard to jurisdictional claims in published maps and institutional affiliations. 